\title{
Distribution System State Estimation Using the Hamiltonian Cycle Theory
}

\author{
Jônatas Boás Leite, Student Member, IEEE, and José Roberto Sanches Mantovani, Member, IEEE
}

\begin{abstract}
Since the origin of energy management systems, state estimation applications have aided in automatic power system operations, mainly for transmission systems. Currently, however, smart grid concepts are modifying the behavior of distribution systems through a rapid increase of controllable distributed generators, demand response, and electric vehicles. Consequently, the advanced metering infrastructure is providing a large amount of synchronized metering data with high accuracy and resolution, which favors the development of state estimation procedures to sustain distribution management systems. Therefore, this paper presents the formulation of a novel algorithm for state estimation solution in distribution networks using the Hamiltonian cycle theory, where the network states are quickly obtained through a calculation scheme under the normal operating conditions.
\end{abstract}

Index Terms-Advanced metering infrastructure (AMI), automatic operations, distribution management system (DMS), Hamiltonian cycle, smart grid, state estimation.

\section{INTRODUCTION}

$\mathbf{S}$ UPERVISORY control and data acquisition (SCADA) systems traditionally connect two environments: 1) the substation; and 2) the operation center [1]. In the beginning, SCADA systems were implanted using a complex electromechanical switching system based on dial telephone technology able to read instruments and operate equipment. The advent of the computer for control and processing transformed SCADA systems into their present form, which allowed for the employment of many advanced applications. As a result, they have been renamed energy management systems (EMSs), and they address state estimation as their main application to provide a complete dataset for the real-time monitoring and control of power systems [2]. Conventional state estimators enable an accurate and efficient monitoring of line flows and bus voltage magnitudes to identify the actual operating state, which can be a normal, emergency or restorative state [3]. A state estimation application typically has the following functions [4], [5].

1) Topology Processor: Defines the system topology via status data of circuit breakers and switches.

Manuscript received September 2, 2014; revised January 13, 2015, March 31, 2015, and June 2, 2015; accepted June 19, 2015. Date of publication July 17, 2015; date of current version December 19, 2015. This work was supported by the São Paulo Research Foundation under Grant 2014/22377-1 and Grant 2013/23590-8, by the Higher Education Personnel Improvement Coordination, and by the National Council for Scientific and Technological Development under Grant 305371/2012-6. Paper no. TSG-00872-2014.

The authors are with the Department of Electrical Engineering, São Paulo State University (UNESP)/FEIS, Ilha Solteira 15385-000, Brazil (e-mail: jonatasboasleite@gmail.com; mant@dee.feis.unesp.br).

Digital Object Identifier 10.1109/TSG.2015.2448940
2) Observability Analysis: Determines the feasibility of state estimation solution for the available measurement set.

3) State Estimation Solution: Calculates the bus voltages and line flows using the available measurement set.

4) Bad Data Processing: Identifies and eliminates the bad data of the available measurement set.

5) Parameter and Structural Error Processing: Estimates various network parameters and detects errors in the network structure.

Since the 1970s, transmission systems have been operated by EMSs, which has motivated the development of different state estimation methods, such as weighted least squares (WLS) [6], least absolute value (LAV) [7] and weighted LAV (WLAV) [8]. Only in the recent past have power utilities implemented distribution automation to enhance service quality, which has resulted in the rise of distribution management systems (DMSs), for which one of the main applications is also state estimation [19]. However, transmission system state estimation methodologies cannot be directly applied to a distribution system because the distribution networks are unbalanced and radial and have a large number of nodes and the impedance ratio $\mathrm{R} / \mathrm{X}$ is larger than or equal to 1 [9]-[21].

The 1990s were marked by the proposition of several techniques for state estimation in distribution systems. In [9], the least square state estimation problem is solved using Kirchhoff's laws alongside the statistical models of loads that represent the distribution transformer loading. Baran and Kelley [10] proposed a basic WLS approach where the simplification of Jacobian matrix elements results in a state estimation strongly dependent on forecasting of the loads. In [11], a branch-current-based state estimator is developed that attempts to incorporate the unbalanced nature of distribution feeders but simplifies the network topology through the pruning of lateral feeders. A current-based formulation is also adopted by [12] where, first, the algorithm estimates the pseudo-measurements in distribution transformers and, subsequently, the nonlinear equations are iteratively solved using Newton's method. Lin and Teng [13] presented a currentbased fast decoupled state estimation in rectangular form; here, the constant gain matrix is decoupled into two matrices. The decoupling is also employed by [14], which introduces the zero injections measurements dealt with via the Lagrange multipliers. Li [15] proposed a stochastic load model influenced by time-varying and weather-related conditions. In [16], a probabilistic model supports the non-normal statistical 
behavior of states and load diversity. Celik and Liu [17] assumed the reliability of the measured data and use a modified version of the Gauss-Seidel load flow algorithm, but this methodology does not support closed meshes and/or dispersed generators (DG).

In 2000s, methodologies of distribution state estimation were evaluated. In this way, Lubkeman et al. [18] performed a field implementation of state estimation and discusses the unsolved issues as the load modeling strategies. Baran [19] concluded that more tailored methods need to be developed in order to address practical problems of distribution operation. Wang and Schulz [20] reviewed the branch-current method and use data from automatic meter reading to estimate the loads on the distribution transformers. Lastly, Singh et al. [21] comparatively assessed the techniques of WLS, WLAV, and Schweppe Huber generalized M. The final results showed that the WLS algorithm works well whenever the noise characteristic is known; for other cases, a new class of algorithms must be developed.

The early 2010s have been influenced by smart grid concepts that promote the integration and evolution of all systems [22]. In the transition to the smart grid, a distribution system requires the implementation of an advanced metering infrastructure (AMI) with distributed command-and-control strategies over all components, geographies and functions of the system. The AMI equipment has a two-way communication channel with the ability to measure and modify customer service-level parameters. An example is the smart meter, which is an essential piece of equipment for the AMI architecture used to identify and measure power consumption. The integration of AMI and DMS is expected to bring promising advancements to various management applications [2]. In this way, Taylor et al. [23] assumed the existence of redundancy in the information data and suggests the development of novel state estimation methods for distribution networks that integrate the DG and smart meters with low-computation time.

This paper follows the smart grid trend and develops a novel state estimation method based on the smart metering system. The proposed method employs a suitable distribution transformer model to couple the state estimation of mediumvoltage (MV) and low-voltage (LV) networks, which allows the creation of a sequence of calculation for each unknown state using the solution of the Hamiltonian cycle problem. In Section II, the proposed state estimation algorithm for the distribution system is described in detail, as well as its mathematical formulation. Numerical results, obtained using the estimator under a real distribution network, are presented and discussed in Section III. Lastly, conclusion and future works are enumerated in Section IV.

\section{State Estimation Algorithm}

Many EMS advanced applications, such as online load flow, load forecast, economic dispatch, stability analysis, security assessment and restoration strategy, employ the data provided by the state estimation algorithm to perform their tasks [4] once the state estimation can determine a reliable set of system voltages through the processing of known network states

$$
\begin{aligned}
& \boldsymbol{\Omega}=\{\omega \mid \omega \in \boldsymbol{J} \cup \boldsymbol{V} \cup \boldsymbol{I}\} \\
& \boldsymbol{\Psi}=\{\psi \mid \psi \in \boldsymbol{\Omega} \curlywedge \omega \text { is measured }\} \\
& \boldsymbol{X}=\{\chi \mid \chi \in \boldsymbol{\Omega} \curlywedge \omega \text { is unknown }\} .
\end{aligned}
$$

In power systems, network states are the sets of bus current injections, $\boldsymbol{J}$, bus voltages, $\boldsymbol{V}$, and branch currents, $\boldsymbol{I}$, whose union produces the set of states, $\boldsymbol{\Omega}$, as given by (1). The smart metering system measures some network states that jointly construct the set of measurements, $\boldsymbol{\Psi}$, formulated by (2). Finally, (3) expresses the set of unknown states, $\boldsymbol{X}$, obtained by the remainder states that complement $\boldsymbol{\Psi}$ to $\boldsymbol{\Omega}$. Thus, state estimation algorithms typically obtain the values of $\boldsymbol{X}$ using the values of $\Psi$ and a sequence of multiple regressions. As an alternative to the traditional approach, the proposed state estimation is performed using the solution of the Hamiltonian cycle problem; however, before introducing its mathematical formulation, some models employed by the proposed approach are developed.

\section{A. Voltage and Current Equations}

The distribution network behavior is linear for certain loading conditions, but its mathematical formulation, which calculates the network states, is nonlinear because the distribution network is modeled using a large and complex electric circuit. Alternately, the distribution network can be seen as an array of many small and simple electrical circuits (network zones) that have linear solutions obtained by Kirchhoff's current laws (KCL) and Kirchhoff's voltage laws (KVL). Table I describes some network zones through the singleline diagram and the mathematical formulation that solves the unknown state for two sweep modes: 1) backward; and 2) forward.

In the backward sweep, the upstream unknown states, $\dot{I}_{U}$ and $\dot{V}_{U}$, can be estimated whenever the set of required states, $\boldsymbol{\Psi}_{\mathbf{1}}\left(\dot{I}_{U}\right)$ or $\boldsymbol{\Psi}_{\mathbf{2}}\left(\dot{V}_{U}\right)$, are known. In (4), the upstream branch current, $\left[\dot{I}_{U}\right]_{3 \times 1}$, is modeled by KCL, and it is equal to the bus current injection, $\left[\dot{J}_{U}\right]_{3 \times 1}$, plus the summation of all currents flowing into the downstream adjacent branches. Equation (5) expresses the model of the upstream bus voltage, $\left[\dot{V}_{U}\right]_{3 \times 1}$, using KVL, where it is equal to a downstream bus voltage, $\left[\dot{V}_{D}\right]_{3 \times 1}$, plus the voltage drop over the downstream adjacent branch.

Similarly, the forward sweep can estimate the unknown downstream states, $\dot{I}_{D}$ and $\dot{V}_{D}$, whenever the set of required states, $\boldsymbol{\Psi}_{\mathbf{3}}\left(\dot{I}_{D}\right), \boldsymbol{\Psi}_{\mathbf{4}}\left(\dot{V}_{D}\right)$ or $\boldsymbol{\Psi}_{\mathbf{5}}\left(\dot{I}_{D}\right)$, are known. In (6), any downstream branch current, $\left[\dot{I}_{D}\right]_{3 \times 1}$, is modeled by KCL, and it is equal to the upstream adjacent branch current, $\left[\dot{I}_{U}\right]_{3 \times 1}$, minus the upstream bus current injection, $\left[\dot{J}_{U}\right]_{3 \times 1}$, minus the summation of all currents flowing into the adjacent branches. Equation (7) expresses the model of the downstream bus voltage, $\left[\dot{V}_{D}\right]_{3 \times 1}$, using $\mathrm{KVL}$, where it is equal to the upstream bus voltage, $\left[\dot{V}_{U}\right]_{3 \times 1}$, minus the voltage drop over its adjacent branch. The downstream branch current, $\left[\dot{I}_{D}\right]_{3 \times 1}$, can also be modeled by (8), where it is equal to 
TABLE I

Single-Line Diagram of the Network Zones and Their Equations to Calculate the Unknown States

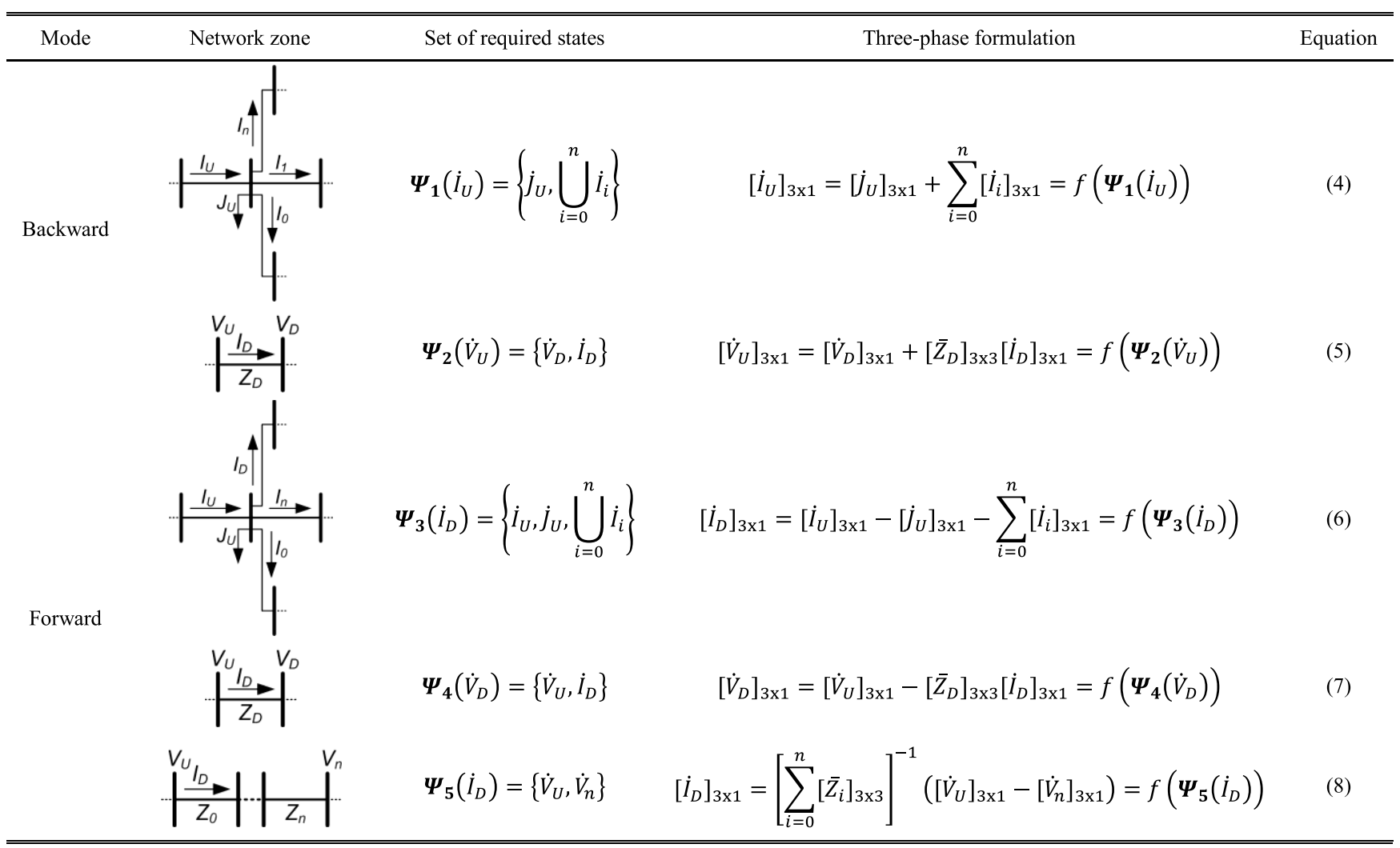

the upstream bus voltage, $\left[\dot{V}_{U}\right]_{3 \times 1}$, minus a remote downstream bus voltage, $\left[\dot{V}_{n}\right]_{3 \times 1}$, and then this voltage drop is divided by the summation of all series-connected impedances between these two buses. This last equation is only used when there is no current injection between the buses with known voltages.

Network zones are discretized elements that have branches and buses from the distribution network. The branches can be distribution lines or transformers. The distribution transformers are typical devices that connect the MV network to the LV network and may cause a phase angle deviation in the voltage and current values according to the connection type. Given this, the mathematical formulations of the network zones must be improved to support the operational characteristics of the distribution transformer.

\section{B. Distribution Transformer Model}

The distribution transformer can be seen as a quadripole, where the parameters of the mathematical formulation are obtained from the information card and short- and open-circuit tests. Fig. 1 shows the electrical diagram of a distribution transformer modeled via the quadripole concepts.

The most common quadripoles, or two-port mathematical models, are referred to as $z-, y-, h-, g_{-}$, and $A B C D$-parameters, and they are described by a 2-by-2 square matrix of complex numbers. The distribution transformer model contains what are referred to as hybrid parameters ( $h$-parameters), and its diagonal $h$-parameters are dimensionless due to the requirement of

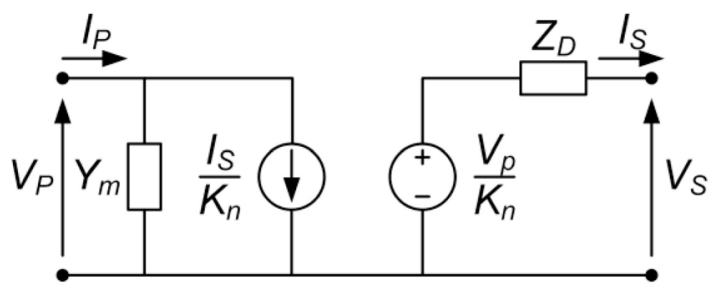

Fig. 1. Analysis of the distribution transformer as quadripole.

various power flow algorithms to calculate the primary current, $\dot{I}_{P}$, on the backward sweep and the secondary voltage, $\dot{V}_{S}$, on the forward sweep

$$
\begin{aligned}
{\left[\begin{array}{l}
\dot{I}_{P} \\
\dot{V}_{S}
\end{array}\right] } & =\left[\begin{array}{cc}
K_{n}^{-1} & \bar{Y}_{m} \\
-\bar{Z}_{D} & K_{n}^{-1}
\end{array}\right]\left[\begin{array}{c}
\dot{I}_{S} \\
\dot{V}_{P}
\end{array}\right] \\
{\left[\begin{array}{l}
{\left[\dot{I}_{P}\right]_{3 \times 1}} \\
{\left[\dot{V}_{S}\right]_{3 \times 1}}
\end{array}\right] } & =\left[\begin{array}{cc}
K_{n}^{-1}[\Phi]_{3 \times 3} & {\left[\bar{Y}_{m}\right]_{3 \times 3}} \\
-\left[\bar{Z}_{D}\right]_{3 \times 3} & K_{n}^{-1}[\Phi]_{3 \times 3}^{T}
\end{array}\right]\left[\begin{array}{c}
{\left[\dot{I}_{S}\right]_{3 \times 1}} \\
{\left[\dot{V}_{P}\right]_{3 \times 1}}
\end{array}\right] .
\end{aligned}
$$

The distribution transformer model per phase is expressed by (9), and its adaptation for calculations in three-phase networks is given by (10). In the distribution transformer model for three-phase networks, a matrix of phase angle deviation, $[\Phi]_{3 \times 3}$, is added. This matrix is the result of the incidence analysis over the transformer terminals for different types of connections. Table II presents the element values of the matrix of phase angle deviation for the main distribution transformer connection types. 
TABLE II

Matrix of Phase ANGLE DeViation for SEVERAL Connection TyPeS

\begin{tabular}{cccccccccc}
\hline \hline Connection & $\phi_{11}$ & $\phi_{12}$ & $\phi_{13}$ & $\phi_{21}$ & $\phi_{22}$ & $\phi_{23}$ & $\phi_{31}$ & $\phi_{32}$ & $\phi_{33}$ \\
\hline${\mathrm{Yg}-\mathrm{Yg}^{\mathrm{a}}}^{\mathrm{a}}$ & 1 & 0 & 0 & 0 & 1 & 0 & 0 & 0 & 1 \\
$\mathrm{Yg}-\Delta^{\mathrm{b}}$ & 1 & 0 & -1 & -1 & 1 & 0 & 0 & -1 & 1 \\
$\Delta-\mathrm{Yg}^{\mathrm{c}}$ & 1 & -1 & 0 & 0 & 1 & -1 & -1 & 0 & 1 \\
$\Delta-\Delta^{\mathrm{d}}$ & 1 & 0 & 0 & 0 & 1 & 0 & 0 & 0 & 1 \\
\hline \hline
\end{tabular}

TABLE III

TRANSFormation RATIO FOR SEVERAL CONNECTION TYPES

\begin{tabular}{cccc}
\hline \hline Connection & $K_{n}$ & Connection & $K_{n}$ \\
\hline${\mathrm{Yg}-\mathrm{Yg}^{\mathrm{a}}}^{\mathrm{a}}$ & $V_{P L} / V_{S L}$ & $\Delta-\mathrm{Yg}^{\mathrm{c}}$ & $\sqrt{3} V_{P L} / V_{S L}$ \\
$\mathrm{Yg}-\Delta^{\mathrm{b}}$ & $V_{P L} / \sqrt{3} V_{S L}$ & $\Delta-\Delta^{\mathrm{d}}$ & $V_{P L} / V_{S L}$ \\
\hline \hline
\end{tabular}

${ }^{\text {a }}$ primary and secondary are connected in grounded wye (Yg);

${ }^{\mathrm{b}}$ primary in grounded wye and secondary in delta connection;

${ }^{c}$ primary in delta and secondary in grounded wye connection;

${ }^{\mathrm{d}}$ primary and secondary are connected in delta $(\Delta)$.

The transformation ratio, $K_{n}$, which is given by the relation between the input voltage and the output voltage, also has its value modified according to the distribution transformer connection type. Table III summarizes the values of $K_{n}$ for the main distribution transformer connection types

$$
\begin{aligned}
& \bar{Z}_{D}=\frac{V_{\mathrm{SL}}^{2}}{S_{3 \varphi}}\left(\frac{P_{\mathrm{Cu}}}{S_{3 \varphi}}+j \sqrt{Z_{\%}^{2}-\left(\frac{P_{\mathrm{Cu}}}{S_{3 \varphi}}\right)^{2}}\right) \\
& \bar{Y}_{m}=\frac{1}{3 V_{\mathrm{PL}}^{2} \sqrt{\frac{1}{P_{\mathrm{Fe}}^{2}}+\left(\frac{\left(1-Z_{\%}\right) V_{\mathrm{SL}}}{2 V_{\mathrm{PL}} S_{3 \varphi}}\right)^{2}}} e^{-\mathrm{jarctan}\left(\frac{2 V_{\mathrm{PL}} S_{3 \varphi}}{P_{\mathrm{Fe}}\left(1-Z_{\%}\right) V_{\mathrm{SL}}}\right) .}
\end{aligned}
$$

The primary line voltage, $V_{\mathrm{PL}}$, and the secondary line voltage, $V_{\mathrm{SL}}$, are values found on the information card of a distribution transformer, where additional values are also found, such as the three-phase power, $S_{3 \varphi}$, and percentage impedance, $Z_{\%}$, which are employed to calculate the dispersion impedance, $\bar{Z}_{D}$, and magnetization admittance, $\bar{Y}_{m}$, as given by (11) and (12). In addition to the values from the information card, the results of the short- and open-circuit tests, such as the copper loss, $P_{\mathrm{Cu}}$, and iron loss, $P_{\mathrm{Fe}}$, are also used to calculate the values of $\bar{Z}_{D}$ and $\bar{Y}_{m}$ that fill the diagonals of the matrices $\left[\bar{Z}_{D}\right]_{3 \times 3}$ and $\left[\bar{Y}_{m}\right]_{3 \times 3}$, respectively.

Once the distribution transformer model was formulated, the Table I equations could be adapted through simple algebraic manipulations to support branches that have a distribution transformer

$$
\begin{aligned}
{\left[\dot{I}_{U}\right]_{3 \times 1} } & =K_{n}^{-1}[\Phi]_{3 \times 3}\left[\dot{I}_{D}\right]_{3 \times 1}+\left[\bar{Y}_{m}\right]_{3 \times 3}\left[\dot{V}_{U}\right]_{3 \times 1} \\
{\left[\dot{V}_{U}\right]_{3 \times 1} } & =K_{n}[\Phi]_{3 \times 3}\left(\left[\dot{V}_{D}\right]_{3 \times 1}+\left[\bar{Z}_{D}\right]_{3 \times 3}\left[\dot{I}_{D}\right]_{3 \times 1}\right) \\
{\left[\dot{I}_{D}\right]_{3 \times 1} } & =K_{n}[\Phi]_{3 \times 3}^{T}\left(\left[\bar{Y}_{m}\right]_{3 \times 3}\left[\dot{V}_{U}\right]_{3 \times 1}+\left[\dot{I}_{U}\right]_{3 \times 1}\right) \\
{\left[\dot{V}_{D}\right]_{3 \times 1} } & =K_{n}^{-1}[\Phi]_{3 \times 3}^{T}\left[\dot{V}_{U}\right]_{3 \times 1}-\left[\bar{Z}_{D}\right]_{3 \times 3}\left[\dot{I}_{D}\right]_{3 \times 1} .
\end{aligned}
$$

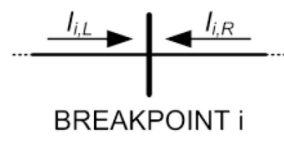

(a)

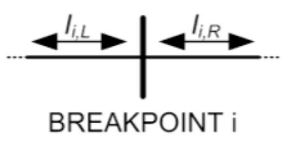

(b)
Fig. 2. Representation of breakpoint. (a) Conventional way. (b) Real behavior.

If the distribution transformer is comparable to a branch, then the upstream states are the primary values and the downstream states are the secondary values of the transformer. In this way, (13) and (14) express the upstream branch current and upstream bus voltage, respectively, for a distribution transformer in the backward sweep mode, while (15) and (16) express the downstream branch current and downstream bus voltage for a distribution transformer in the forward sweep mode. In summary, the quadripole transformer model fully operates in the backward/forward sweep mode without the matrices inversion eliminating singularity problem.

\section{Meshed Distribution Networks}

The transformer is not the unique particularity of electrical networks, e.g., meshed distribution networks have special buses named breakpoints that close the loops; thus, the division of a meshed network into many network zones creates network zones with two branch currents converging to one breakpoint because the direction of branch currents is given in the conventional way [see Fig. 2(a)].

In the conventional way, the network zone of the breakpoint has two opposite currents, one from the left, $\left[\dot{I}_{i, L}\right]_{3 \times 1}$, and another from the right, $\left[\dot{I}_{i, R}\right]_{3 \times 1}$, which does not correspond to the real behavior because the breakpoint is a passing bus instead of a drain bus. Indeed, the directions of currents are unknown, as in Fig. 2(b)

$$
\left[\dot{I}_{U}\right]_{3 \times 1}=\left[\dot{I}_{i, L}\right]_{3 \times 1}=\left[\dot{I}_{i, R}\right]_{3 \times 1}=\left[\dot{I}_{D}\right]_{3 \times 1} .
$$

Equality (17) is essential to preserve the robustness of Table I's equations. In the backward sweep, the upstream unknown states are estimated by the downstream known states, thereby, from the left branch's perspective, $\left[\dot{I}_{i, L}\right]_{3 \times 1}$ is the upstream unknown state and is equal to $\left[\dot{I}_{i, R}\right]_{3 \times 1}$, which is the downstream known state, and, from the right branch's perspective, a similar correspondence is used.

In the network zone of the breakpoint, the directions of the branch currents are unknown, as is the estimation model of each unknown state; therefore, a search procedure is needed to determine the estimation model of each unknown state and the instant when each unknown state should be calculated.

\section{Hamiltonian Cycle Problem}

In mathematics, a graph represents a set of points (vertices) and lines (edges) where each line connects two points. A particular graph type is the Hamiltonian graph, which has one or more Hamiltonian cycles where each vertex is visited only once according to a Hamiltonian path [24], [25]. If the elements of $\boldsymbol{X}$ are disposed in a Hamiltonian graph where each vertex assumes one element $\chi_{i}$, i.e., each vertex takes an unknown state, then there must be a suitable 
Hamiltonian path able to provide a sequence of calculation of the unknown states. In this way, one of the main objectives of the proposed procedure of distribution system state estimation is the solution of the Hamiltonian cycle problem in a manner that can be formulated as an integer linear programming problem

$$
\min F(\boldsymbol{X})=\sum_{j=1}^{n_{X}} C_{j}
$$

subject to

$$
\begin{aligned}
& \sum_{i=1, i \neq j}^{n_{X}} h_{i j}=1, \quad j=1,2, \ldots, n_{X} \\
& \sum_{\substack{n_{X} \\
n_{i}}} h_{i j}=1, \quad i=1,2, \ldots, n_{X} \\
& s_{i}-s_{j}+n_{X} h_{i j} \leq n_{X}-1, \quad s_{i} \neq n_{X}, \quad i, j=1,2, \ldots, n_{X}
\end{aligned}
$$

$$
\boldsymbol{A}(j)=\left\{\chi_{i} \mid s_{i}<s_{j}\right\}, \quad i, j=1,2, \ldots, n_{X}
$$$$
C_{j}=\left\{\begin{array}{ll}
0, & \text { if } \boldsymbol{\Psi}_{k}\left(\chi_{j}\right) \subset \boldsymbol{A}(j) \cup \boldsymbol{\Psi} \\
1, & \text { otherwise }
\end{array}, \quad j=1,2, \ldots, n_{X}\right.
$$

$$
1 \leq s_{i} \neq s_{j} \leq n_{X}, \quad i, j=1,2, \ldots, n_{X}
$$

$h_{i j}, C_{j} \in\{0,1\}$

$s_{i} \in Z$.

In (18), $F(\boldsymbol{X})$ is the objective function of the proposed Hamiltonian cycle problem, and it has an optimal value equal to zero when the suitable Hamiltonian cycle is found by the optimization process that uses the restrictions from (19) to (24) as the pathfinder. Equations (19) and (20) are typical constraints of the travelling salesman problem, and they aid in the creation of arbitrary cycles. A square binary matrix $[H]_{n_{X} \times n_{X}}$ can represent these arbitrary cycles of the graph with $n_{X}$ vertices, where the binary element $h_{i j}$ is equal to 1 whenever there is an edge oriented from the vertex of $\chi_{i}$ to the vertex of $\chi_{j}$ and equal to 0 when there is not such an edge. Constraint (19) avoids the repetition of the value 1 in the matrix rows, while (20) avoids such repetition in the matrix columns. In this way, each vertex is visited only once by one or more disjoint cycles.

Constraint (21) forces the construction of the Hamiltonian cycle; for example, Fig. 3(c) shows the matrix $[H]_{6 \times 6}$ for the two disjoint cycles illustrated in Fig. 3(a). Despite the compliance with (19) and (20), this matrix is infeasible because it contradicts (21), i.e., $s_{3}-s_{2}+n_{X} h_{32}=8$ is not less than or equal to $n_{X}-1=5$. Alternately, Fig. 3(d) demonstrates a feasible matrix that represents the Hamiltonian cycle shown in Fig. 3(b). The variable $s_{i}$ is the cycle step and indicates the instant when the vertex of $\chi_{i}$ is visited; consequently, the set $\boldsymbol{A}(j)$ contains all visited vertices until the visit to the vertex of $\chi_{j}$, as given by (22). In other words, if the estimation model, $f\left(\boldsymbol{\Psi}_{k}\left(\chi_{j}\right)\right)$, of the unknown state is obtained during the visit to its vertex, then the set $\boldsymbol{A}(j)$ accumulates all of the unknown states whose values were already modeled in a previous visit. In this way, (23) expresses the cost

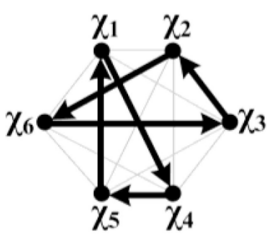

(a)

(c)

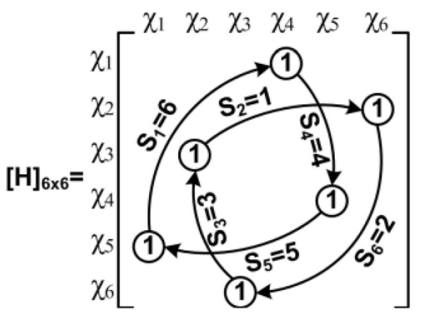

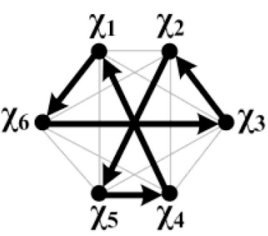

(b)

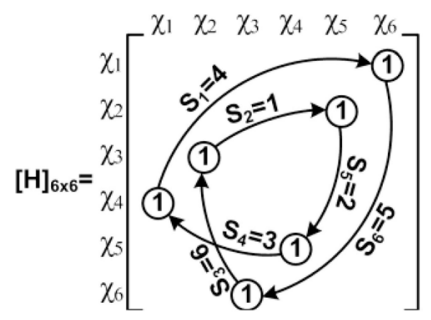

(d)
Fig. 3. Construction of the binary matrix $[H]_{6 \times 6}$. (a) Two disjoint cycles. (b) Hamiltonian cycle. Matrix representation of (c) disjoint cycles and (d) Hamiltonian cycle.

of a visit to vertex of $\chi_{j}$, and this cost, $C_{j}$, is equal to zero whenever the set of required states, $\boldsymbol{\Psi}_{\boldsymbol{k}}\left(\chi_{j}\right)$, is a subset of the union of sets $\boldsymbol{A}(j)$ and $\boldsymbol{\Psi}$. Table I establishes five basic estimation models and their sets of required states, $\Psi_{1}\left(\dot{I}_{U}\right)$, $\boldsymbol{\Psi}_{\mathbf{2}}\left(\dot{V}_{U}\right), \boldsymbol{\Psi}_{\mathbf{3}}\left(\dot{I}_{D}\right), \boldsymbol{\Psi}_{\mathbf{4}}\left(\dot{V}_{D}\right)$, and $\boldsymbol{\Psi}_{\mathbf{5}}\left(\dot{I}_{D}\right)$, for each network zone; however, these sets can vary according to the network zone components. Thus, the original set $\Psi_{1}\left(\dot{I}_{U}\right)$ is altered to $\Psi_{\mathbf{1}}^{\mathbf{B P}}\left(\dot{I}_{U}\right)=\left\{\dot{I}_{D}\right\}$ when the network zone has a breakpoint and to $\boldsymbol{\Psi}_{1}^{\mathrm{DT}}\left(\dot{I}_{U}\right)=\left\{\dot{I}_{D}, \dot{V}_{D}\right\}$ in the presence of a distribution transformer that also modifies the original set $\boldsymbol{\Psi}_{\mathbf{3}}\left(\dot{I}_{D}\right)$ to $\boldsymbol{\Psi}_{\mathbf{3}}^{\mathbf{D T}}\left(\dot{I}_{D}\right)=\left\{\dot{I}_{U}, \dot{V}_{U}\right\}$

The cycle step, $s_{i}$, is an integer that varies, without repetition, from one to the number of unknown states, $n_{X}$, as given by (24). Lastly, the edge that closes the Hamiltonian cycle ( $\left.s_{i}=n_{X}\right)$ is removed to produce the Hamiltonian path, i.e., the sequence of calculation of the unknown states.

\section{E. Hamiltonian Cycle via Specialized Algorithm}

The Hamiltonian cycle problem is an NP-hard problem, and, as such, it requires a high-computational cost to find any Hamiltonian cycle without violating the constraints from (19) to (21) when the search space is large. The reduction of the search space is an efficient approach that dramatically decreases the computational processing time, allowing for the application of the proposed methodology for real-time control. Fig. 4 shows the flowchart of a specialized algorithm where an auxiliary cycle is swept downward and upward during the searching procedure for a suitable Hamiltonian cycle.

First, the algorithm arranges the elements of the set $\boldsymbol{X}$ within the auxiliary cycle; thereby, more peripheral state variables stay at the end of the auxiliary cycle, whereas the state variables near the substation stay at the beginning of the auxiliary cycle. Second, the initialization is performed, e.g., the sweep is set to downward and all values of $C_{j}$ are set to 1 . Subsequently, the algorithm starts the procedure for the construction of the Hamiltonian cycle using (22) and (23). If the value of $C_{j}$ is 


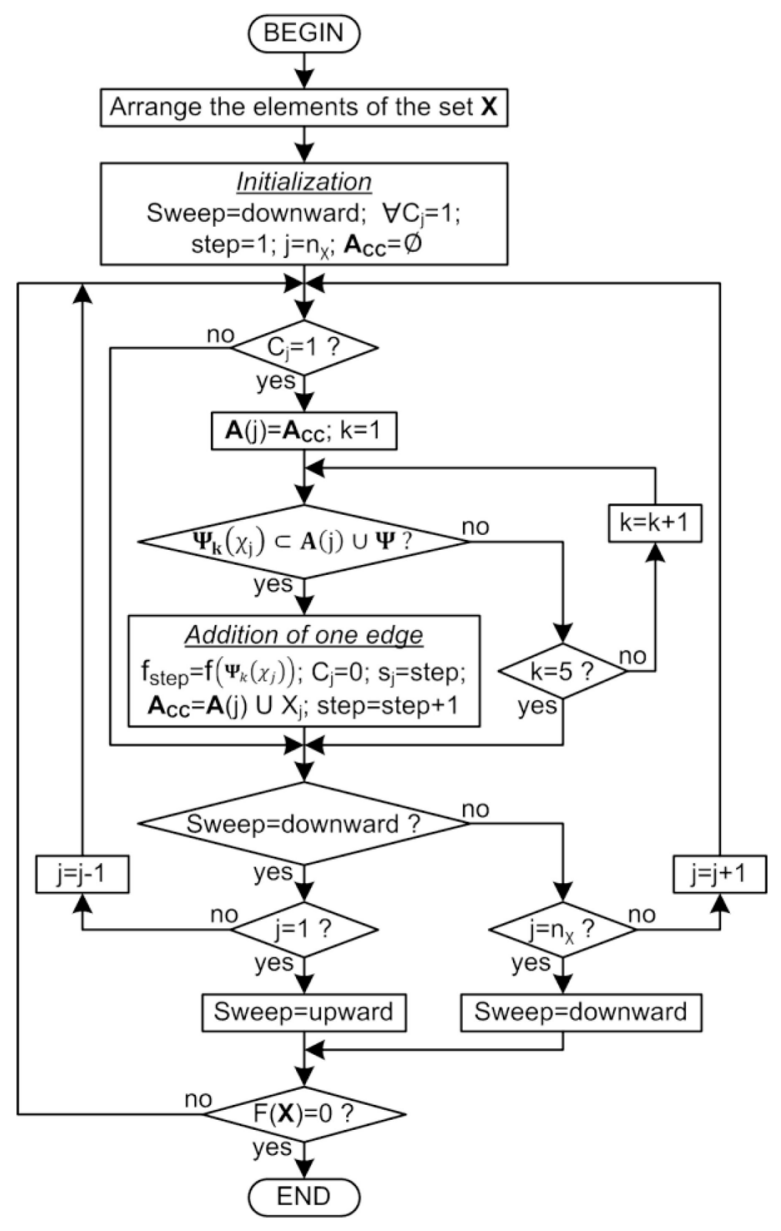

Fig. 4. Flowchart of the algorithm used to find the Hamiltonian cycle.

equal to 1 , the vertex of $\chi_{j}$ is not included in the Hamiltonian cycle under construction, and then the set $\boldsymbol{A}(j)$ is built and the possibility of modeling the value of $\chi_{j}$ is verified. In the case of confirmation of the possibility, the addition of one edge to the Hamiltonian cycle under construction is performed. In other words, the estimation model $f\left(\Psi_{\boldsymbol{k}}\left(\chi_{j}\right)\right)$ is specified and attributed to $f_{\text {step }}$, the value of $C_{j}$ goes to zero and $s_{i}$ assumes the value of the cycle step, which is also incremented by one unit. These procedures are repeated by the algorithm throughout the entire downward sweep. Thereafter, the minimization of $F(\boldsymbol{X})$ is verified, i.e., if its value is equal to zero, then the Hamiltonian cycle under construction is concluded, and the algorithm stops the construction process; otherwise, a new upward sweep is started to complete the Hamiltonian cycle. The algorithm continues performing new sweeps until it completes the Hamiltonian cycle.

The specialized algorithm used to find the suitable Hamiltonian cycle is a routine of the full procedure of state estimation, as shown by Fig. 5, where the solution of the optimization problem is only performed when the distribution system is alarmed due to a change of topology or a fault of the AMI. Under normal operating conditions, state estimation is rapidly performed via the Hamiltonian path, where an updated set of measurements is employed to calculate each value of $f_{\text {step }}$, i.e., the estimation of each unknown state.

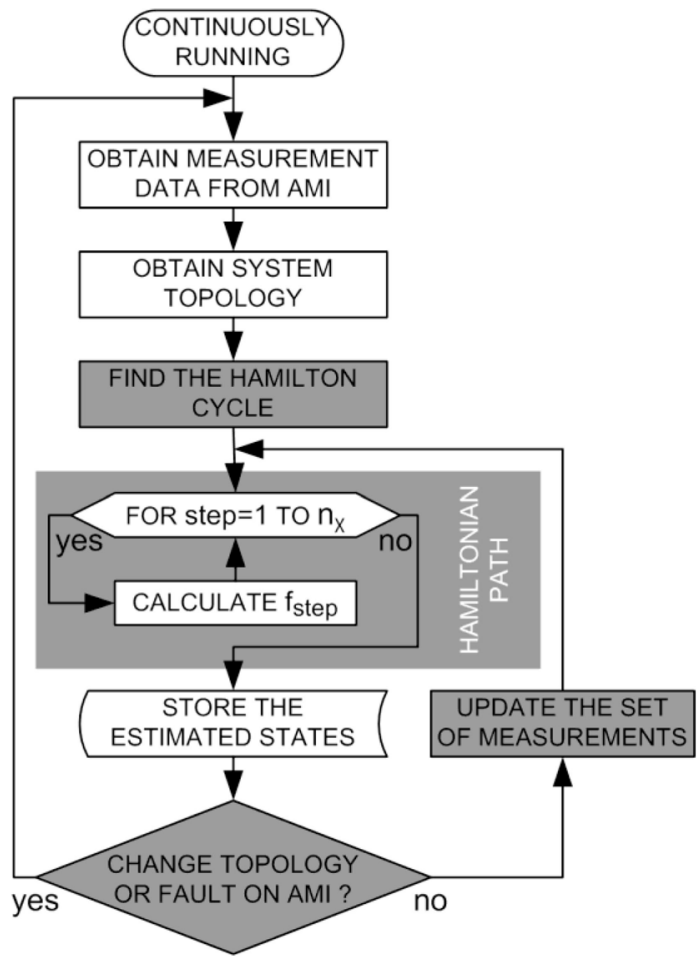

Fig. 5. Flowchart of the proposed procedure of state estimation.

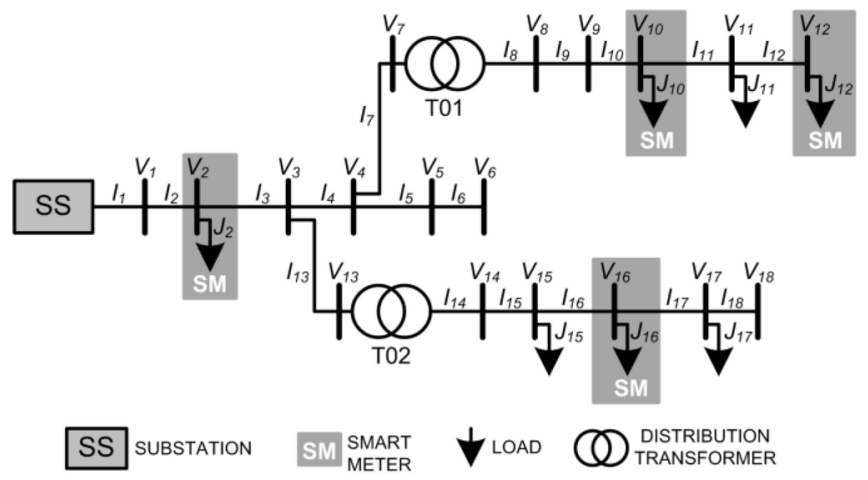

Fig. 6. Topological diagram of the simplified distribution network.

\section{F. Case Study: Simplified Distribution Network}

The case study illustrates the execution of the specialized algorithm over a simplified distribution network that has a satisfactory number of electrical devices and a realistic topology. Furthermore, the redundancy of the AMI infrastructure is broken, and only some loads are metered.

Fig. 6 shows the diagram of the simplified network that allows for the construction of the set of measurements, $\Psi=$ $\left\{\dot{V}_{2}, \dot{I}_{2}, \dot{V}_{10}, \dot{I}_{10}, \dot{V}_{12}, \dot{I}_{12}, \dot{V}_{16}, \dot{I}_{16}\right\}$, whose size is $n_{\Psi}=$ 8. Analogously, it is possible construct the set of unknown states, $\boldsymbol{X}$, whose size is $n_{X}=35$.

The states are indexed in concordance to the network topology. In this way, the states enter sequentially into the auxiliary cycle $\left(\chi_{1}=\dot{J}_{1}, \chi_{2}=\dot{V}_{1}, \ldots, \chi_{35}=\dot{V}_{18}\right)$, as shown by Fig. 7. The downward sweep scans the unknown states from $\chi_{35}$ to $\chi_{1}$, while the upward sweep scans the unknown states from $\chi_{1}$ to $\chi_{35}$ in order to find the estimation model of 


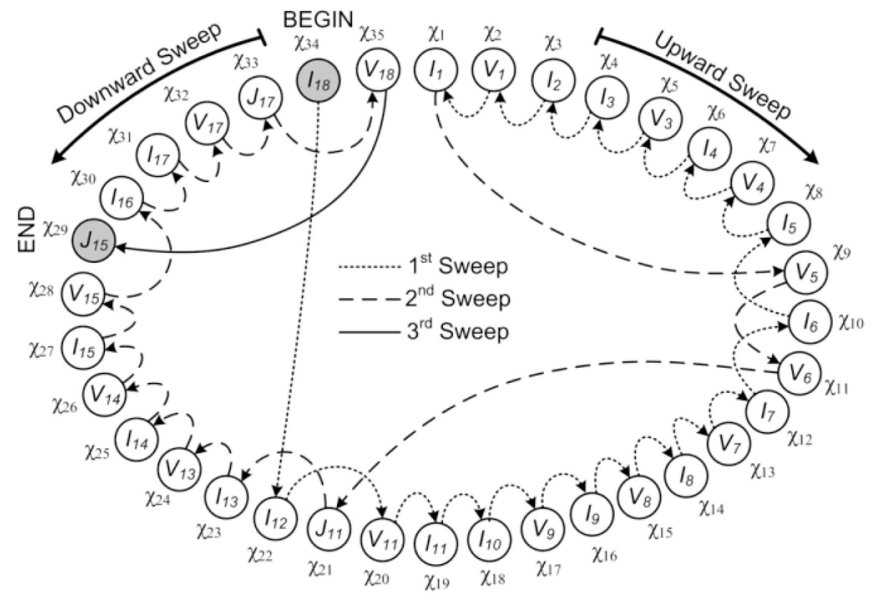

Fig. 7. Hamiltonian path obtained by the specialized algorithm.

TABLE IV

Sequence of Calculation Obtained From the Hamiltonian Path

\begin{tabular}{|c|c|c|c|}
\hline$s_{j}$ & $f\left(\boldsymbol{\Psi}_{\boldsymbol{k}}\left(\chi_{j}\right)\right)$ & $s_{j}$ & $f\left(\boldsymbol{\Psi}_{k}\left(\chi_{j}\right)\right)$ \\
\hline 1 & {$\left[\dot{I}_{18}\right]=[\dot{0}]$} & & {$\left[\dot{V}_{1}\right]=\left[\dot{V}_{2}\right]+\left[\bar{Z}_{1,2}\right]\left[\dot{I}_{2}\right]$} \\
\hline 2 & {$\left[\dot{I}_{12}\right]=\left[\dot{j}_{12}\right]$} & 20 & {$\left[\dot{i}_{1}\right]=\left[\dot{I}_{2}\right]$} \\
\hline 3 & {$\left[\dot{V}_{11}\right]=\left[\dot{V}_{12}\right]+\left[\bar{Z}_{11,12}\right]\left[\dot{I}_{12}\right]$} & 21 & {$\left[\dot{V}_{5}\right]=\left[\dot{V}_{4}\right]-\left[\bar{Z}_{4,5}\right]\left[\dot{I}_{5}\right]$} \\
\hline 4 & {$\left[\dot{I}_{11}\right]=\left[\bar{Z}_{10,11}\right]^{-1}\left(\left[\dot{V}_{10}\right]-\left[\dot{V}_{11}\right]\right)$} & 22 & {$\left[\dot{V}_{6}\right]=\left[\dot{V}_{5}\right]-\left[\bar{Z}_{5,6}\right]\left[\dot{I}_{6}\right]$} \\
\hline 5 & {$\left[\dot{I}_{10}\right]=\left[\dot{j}_{10}\right]+\left[\dot{I}_{11}\right]$} & 23 & {$\left[\dot{j}_{11}\right]=\left[\dot{I}_{11}\right]-\left[\dot{i}_{12}\right]$} \\
\hline 6 & {$\left[\dot{V}_{9}\right]=\left[\dot{V}_{10}\right]+\left[\bar{Z}_{9,10}\right]\left[\dot{I}_{10}\right]$} & 24 & {$\left[\dot{I}_{13}\right]=\left[\dot{I}_{3}\right]-\left[\dot{I}_{4}\right]$} \\
\hline 7 & {$\left[\dot{I}_{9}\right]=\left[\dot{I}_{10}\right]$} & 25 & {$\left[\dot{V}_{13}\right]=\left[\dot{V}_{3}\right]-\left[\bar{Z}_{3,13}\right]\left[\dot{I}_{13}\right]$} \\
\hline 8 & {$\left[\dot{V}_{8}\right]=\left[\dot{V}_{9}\right]+\left[\bar{Z}_{8,9}\right]\left[\dot{I}_{9}\right]$} & 26 & {$\left[\dot{1}_{14}\right]=k_{n}[\Phi]^{T}\left(\left[\bar{Y}_{m}\right]\left[\dot{V}_{13}\right]+\left[\dot{I}_{13}\right]\right)$} \\
\hline 9 & {$\left[\dot{I}_{8}\right]=\left[\dot{I}_{9}\right]$} & 27 & {$\left[\dot{V}_{14}\right]=k_{n}^{-1}[\Phi]^{T}\left[\dot{V}_{13}\right]+\left[\bar{Z}_{D}\right]\left[\dot{I}_{14}\right]$} \\
\hline 10 & {$\left[\dot{V}_{7}\right]=k_{n}[\Phi]\left(\left[\dot{V}_{8}\right]+\left[\bar{Z}_{D}\right]\left[\dot{I}_{8}\right]\right)$} & 28 & {$\left[\dot{I}_{15}\right]=\left[\dot{I}_{14}\right]$} \\
\hline 11 & {$\left[\dot{I}_{7}\right]=k_{n}^{-1}[\Phi]\left[\dot{I}_{8}\right]+\left[\bar{Y}_{m}\right]\left[\dot{V}_{7}\right]$} & 29 & {$\left[\dot{V}_{15}\right]=\left[\dot{V}_{14}\right]-\left[\bar{Z}_{14,15}\right]\left[\dot{I}_{15}\right]$} \\
\hline 12 & {$\left[\dot{I}_{6}\right]=[\dot{0}]$} & 30 & {$\left[\dot{I}_{16}\right]=\left[\bar{Z}_{15,16}\right]^{-1}\left(\left[\dot{V}_{15}\right]-\left[\dot{V}_{16}\right]\right)$} \\
\hline 13 & {$\left[\dot{I}_{5}\right]=\left[\dot{i}_{6}\right]$} & 31 & {$\left[\dot{i}_{17}\right]=\left[\dot{I}_{16}\right]-\left[\dot{j}_{16}\right]$} \\
\hline 14 & {$\left[\dot{V}_{4}\right]=\left[\dot{V}_{7}\right]+\left[\bar{Z}_{4,7}\right]\left[\dot{I}_{7}\right]$} & 32 & {$\left[\dot{V}_{17}\right]=\left[\dot{V}_{16}\right]-\left[\bar{Z}_{16,17}\right]\left[\dot{I}_{17}\right]$} \\
\hline 15 & {$\left[\dot{I}_{4}\right]=\left[\dot{I}_{7}\right]+\left[\dot{I}_{5}\right]$} & 33 & {$\left[\dot{J}_{17}\right]=\left[\dot{I}_{17}\right]-\left[\dot{I}_{18}\right]$} \\
\hline 16 & {$\left[\dot{V}_{3}\right]=\left[\dot{V}_{4}\right]+\left[\bar{Z}_{3,4}\right]\left[\dot{I}_{4}\right]$} & 34 & {$\left[\dot{V}_{18}\right]=\left[\dot{V}_{17}\right]-\left[\bar{Z}_{17,18}\right]\left[\dot{I}_{18}\right]$} \\
\hline 17 & {$\left[\dot{I}_{3}\right]=\left[\bar{Z}_{2,3}\right]^{-1}\left(\left[\dot{V}_{2}\right]-\left[\dot{V}_{3}\right]\right)$} & 35 & {$\left[\dot{j}_{15}\right]=\left[\dot{I}_{15}\right]-\left[\dot{1}_{16}\right]$} \\
\hline 18 & {$\left[\dot{I}_{2}\right]=\left[\dot{I}_{3}\right]+\left[\dot{j}_{2}\right]$} & & \\
\hline
\end{tabular}

each $\chi_{j}$. In each sweep, the algorithm constructs a section of the Hamiltonian cycle; for example, Fig. 7 shows the sections constructed by the first, second, and third sweeps. The final product is the Hamiltonian path that is a sequence of calculation for each unknown state through the found estimation model, as given by Table IV.

\section{Assessment of the State Estimation}

The distribution network in [26], which was used to assess the performance of the proposed state estimation algorithm, is a real network and has an MV level equal to $13.8 \mathrm{kV}$ and an LV level of $220 \mathrm{~V}$. Fig. 8 summarizes the network topology of several electrical devices, such as loads, distribution transformers, capacitors, distributed generators, and switches. The reduced topological diagram includes one LV network, but each distribution transformer has a downstream LV network. The entire real distribution network has 3756 states, of which 1016 states are measured and 2740 states are unknown. The measurement of states was performed using 508 smart meters installed initially in each load of the testing system.

The state estimation procedure has two conditions that require the creation of a new sequence of calculation: 1) a change of topology; and 2) a fault of the AMI. The first condition occurs during restoration procedures where switches are opened and closed to clean a fault of the distribution network, changing the original topology of the network. In the second condition, the AMI architecture cannot measure some network states because the communication system or a certain quantity of smart meters is not working perfectly. In this way, the assessment procedure should chiefly verify the state estimation performance for these two conditions.

\section{A. Change of Topology}

The testing distribution network has a radial topology when the switches SW1 and SW2 are opened. Fig. 9 shows a dispersion chart that indicates errors of voltage and current in each bus of the radial network. The errors were obtained by the absolute value of the difference between estimated states and states collected from a smart grid simulation environment [27], i.e., $\left|I_{i}^{\mathrm{SE}}-I_{i}^{\mathrm{PF}}\right|$ for current and $\left|V_{i}^{\mathrm{SE}}-V_{i}^{\mathrm{PF}}\right|$ for voltage. The dispersion chart demonstrates that the current errors reached a maximum value of $40 \times 10^{-8} \mathrm{~A}$ while voltage errors achieved a maximum value of $25 \times 10^{-8} \mathrm{~V}$ for all buses of the distribution network.

The change of the topology of the test network was made by closing switches SW1 and SW2, which created two loops and changed the network topology from radial to meshed. This meshed network requested a new Hamiltonian path to estimate the unknown states. Fig. 10 shows the performance of the state estimation procedure for the meshed network. In comparison to the performance for the radial network, the dispersion chart demonstrates a slight difference of the state estimation performance because of the utilization of a different Hamiltonian path constructed using the addition of (17). The state estimation again achieved errors of voltage and current of the order $10^{-8}$.

The previous results confirm the immunity of the state estimation algorithm to the change of topology, showing that the proposed methodology works well for a smart grid that has a meshed network. Furthermore, the results also demonstrated that the behavior of other electrical devices, such as capacitors, distributed generators, and distribution transformers, did not influence the performance of the proposed methodology of state estimation.

The fluctuation of the power consumption throughout the day is an important characteristic of the electrical loads and determines the behavior of the distribution networks. The assessment of the influence of load fluctuation on the state estimation method is needed to guarantee its efficiency. Fig. 11 shows a dispersion chart that was built by the errors of 


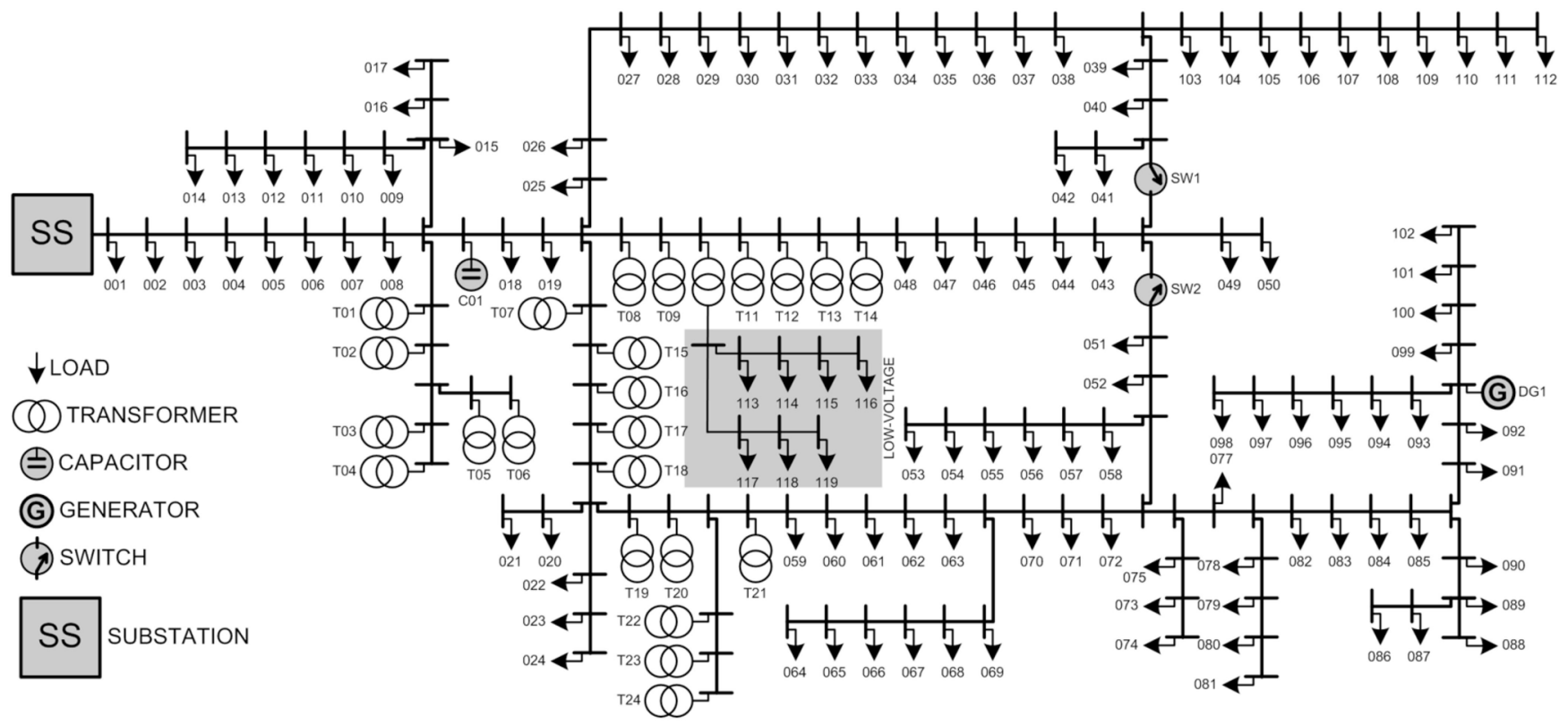

Fig. 8. Topological diagram of real distribution network that was used to assess the state estimation.

\section{Estimation error in the radial network}

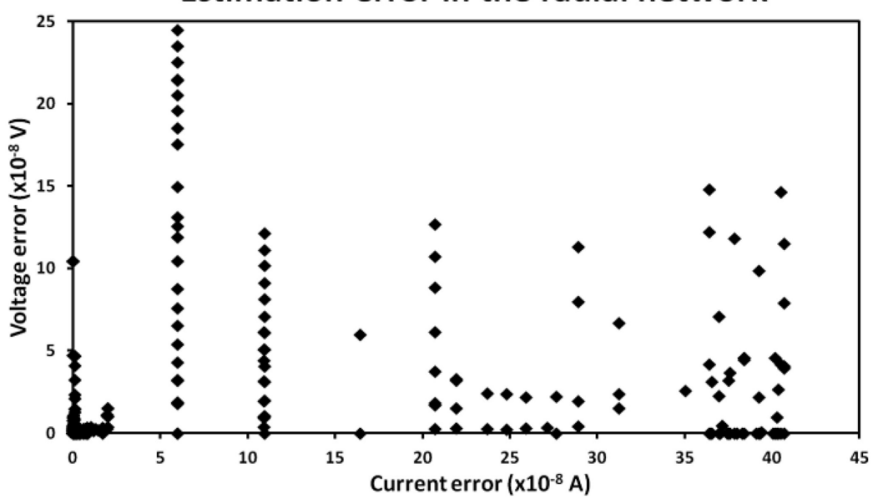

Fig. 9. Performance of the state estimation for the radial network.

apparent power of each bus of the distribution network over the hours of a day. Similar to the errors of voltage and current, the differences of the apparent power remained small. Moreover, more drastic variations occurred between 2 and $4 \mathrm{~h}$ when the apparent power error achieved values of approximately $20 \times 10^{-3}$ VA. Fortunately, observed variations were not sufficiently large to impact the performance of the distribution system state estimation.

Until now, the distribution system state estimation algorithm was assessed against an ideal metering system by which values of voltage and current are measured without errors. In [26], the simulation platform permits the emulation of smart meters through the addition of the quantization noise to the monitored electrical parameters. Fig. 12 shows the performance of the state estimation when the quantization noise is added to all measurements.

The quantization noise had a seemingly uniform distribution over the interval of $-23 \times 10^{-5}$ to $23 \times 10^{-5}$ p.u. of apparent power error, where the greatest frequencies of the estimation error also occurred, as expected. However, the estimation error

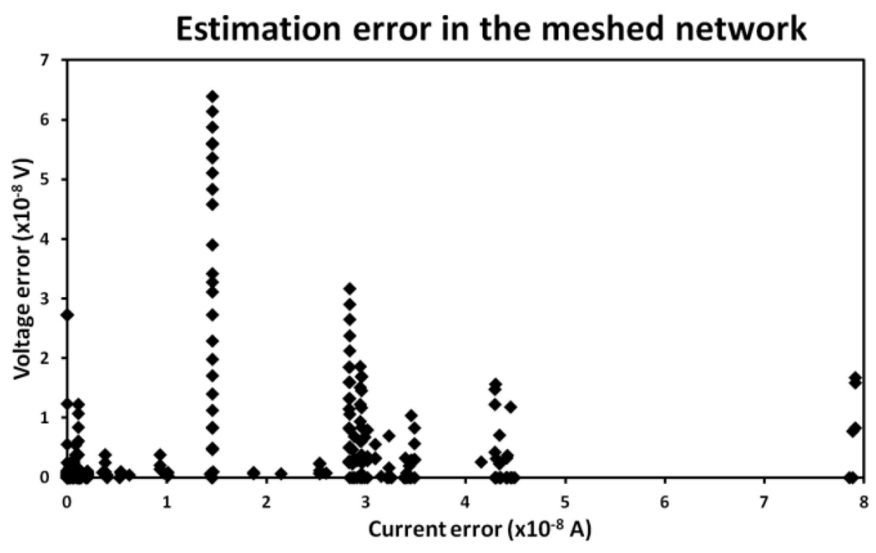

Fig. 10. Performance of the state estimation for the meshed network.

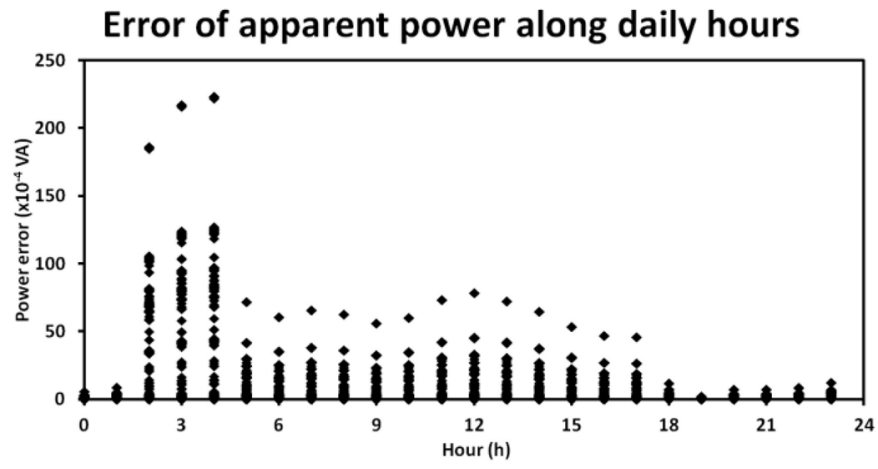

Fig. 11. Performance of the state estimation throughout the day.

propagated beyond this error interval; fortunately, the occurrence frequencies were small and decreased with the increase of the apparent power error. This influence of quantization noise on the state estimation procedure was quantitatively measured through the calculation of common performance metrics, 


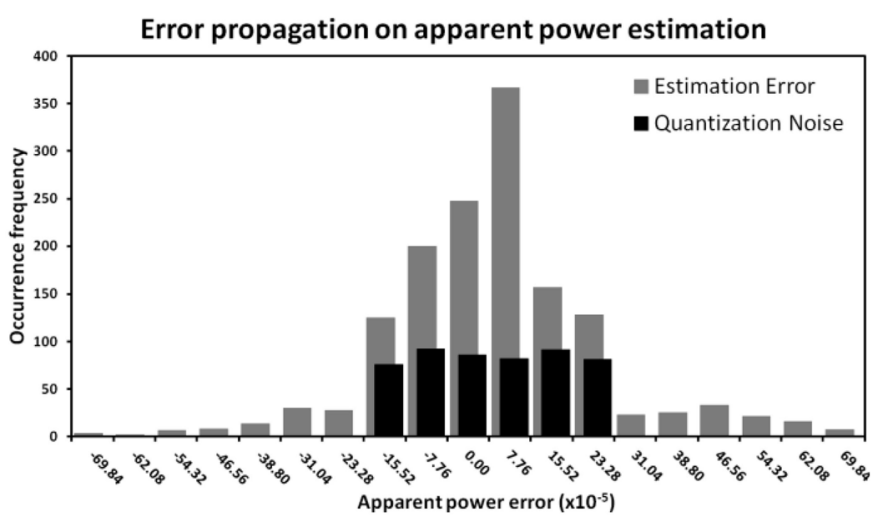

Fig. 12. State estimation performance for the addition of quantization noise.

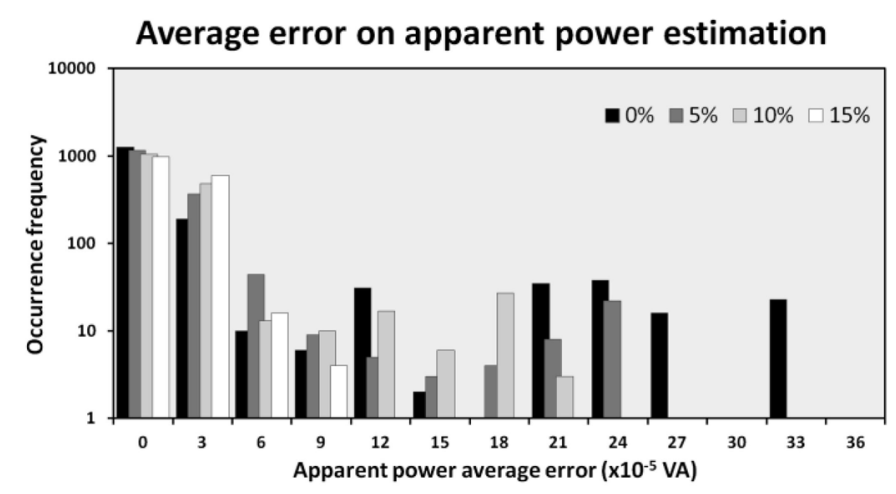

Fig. 13. Performance of the state estimation under a fault of the AMI.

such as average error, $\mu=7.17 \times 10^{-5}$, and error variance, $\sigma^{2}=6.21 \times 10^{-3}$. Furthermore, the calculation of the mean absolute error, MAE $=16.48 \times 10^{-5}$, revealed how close the estimated states were to the simulated states.

Regarding gross errors, the state estimation application must have a bad data processing ability to detect and eliminate these erroneous measurements. The measurement elimination behaves as a fault of the AMI that requires the construction of a new Hamiltonian cycle because a portion of the measured states is lost and this increases the size of the set of unknown states.

\section{B. Fault of the AMI}

Measurement faults are consequences of data loss due to failures of the communication system or the bad operation of smart meters. The assessment of the robustness evaluates the capability of the state estimation algorithm to continue operation even after measurement loss. The condition of measurement loss was simulated by the removal of a certain percentage of smart meters from the distribution network. Fig. 13 presents the results of the simulation of measurement loss for the removal of $0 \%, 5 \%, 10 \%$, and $15 \%$ of the smart meters. The histogram of the apparent power average error compares the performance of the state estimation algorithm for different scenarios of simulation. The state estimation procedure converged in all of the simulation scenarios, and the greatest occurrence frequencies were in the range from 0 to $3 \times 10^{-5}$ of the average error. In the scenario of the
TABLE V

COMPUTATIONAL Cost (ms)

\begin{tabular}{ccc}
\hline \hline Measurement loss & $\begin{array}{c}\text { Time to find the } \\
\text { Hamiltonian cycle }\end{array}$ & $\begin{array}{c}\text { Time to estimate the } \\
\text { network states }\end{array}$ \\
\hline $0 \%$ & 51.2264 & 1.4925 \\
$5 \%$ & 51.6778 & 1.5307 \\
$10 \%$ & 52.0648 & 1.5831 \\
$15 \%$ & 52.0871 & 1.6886 \\
\hline \hline
\end{tabular}

removal of $15 \%$ of the smart meters, only $22 \%$ of all states were known.

The computational cost is another important assessment criterion for any state estimation algorithm; thus, the processing time was measured for the different scenarios of measurement loss that can influence the state estimation performance. The computational environment employed to measure the processing time is an Intel Core 2 Duo CPU running at $2.0 \mathrm{GHz}$ with $2.0 \mathrm{~GB}$ of RAM. The time to find the Hamiltonian cycle slightly increased from 51.23 to 52.09 ms over all scenarios, whereas the time to estimate the network states increased by $200 \mu \mathrm{s}$ for the same assessment interval, as presented by Table V. The results of the computational cost highlight the rapidness of the proposed state estimation procedure, as it spends only $1.5 \mathrm{~ms}$ to calculate the network states under normal operating conditions, which are predominant and represent more than $90 \%$ of the operation time.

\section{CONCLUSION}

The pseudo-measurement, used to forecast the distribution transformer loads, is the major error source of the traditional distribution state estimation methods. The proposed state estimation algorithm does not require statistical models to estimate the nonmeasured loads and is also designed to operate the MV networks in conjunction with LV networks, as established by the smart grid concepts that stimulate the introduction of consumers in the operation of the distribution grid. Moreover, the proposed methodology only uses the iterative algorithm to find the Hamiltonian cycle under the contingency condition, which is relatively a short time interval, in comparison to the normal condition, when the network states are obtained by direct calculations with low computational cost, producing a quick state estimation that is ideal for smart grid operations.

The numerical results demonstrated the immunity of the proposed state estimation procedure to network topology change and its compatibility with several types of electrical devices of the distribution network. The addition of quantization noise demonstrated the error propagation and its reduced impact on the state estimation. Additionally, the simulations of fault of the AMI architecture showed that the proposed state estimation procedure remained working with small errors even after the loss of $15 \%$ of measurements. In this way, the state estimation also demonstrated immunity to faults of the AMI architecture, which should be an essential requirement for smart grid operation due to the deep integration between the power and communication systems. 


\section{REFERENCES}

[1] Substation Automation Tutorial, 1st ed., Piscataway, NJ, USA: IEEE PES, 2003, pp. 1-23.

[2] Z. Li et al., "Toward smart distribution management by integrating advanced metering infrastructure," Elect. Power Syst. Res., vol. 105, no. 1, pp. 51-56, Dec. 2013.

[3] A. Monticelli, State Estimation in Electric Power Systems: A Generalized Approach. New York, NY, USA: Springer, 1999.

[4] M. Shahidehpour and Y. Wang, Communication and Control in Electric Power Systems: Application of Parallel and Distributed Processing, 1st ed. Hoboken, NJ, USA: Wiley, 2003.

[5] A. Abur and A. G. Expósito, Power System State Estimation: Theory and Implementation, 1st ed. Boca Raton, FL, USA: CRC Press, 2004.

[6] A. Bose and K. A. Clements, "Real-time modeling of power networks," Proc. IEEE, vol. 75, no. 12, pp. 1607-1622, Dec. 1987

[7] W. W. Kotiuga, "Development of a least absolute value power system tracking state estimator," IEEE Trans. Power App. Syst., vol. PAS-104, no. 5, pp. 1160-1166, May 1985.

[8] M. K. Celik and A. Abur, "A robust WLAV state estimator using transformations," IEEE Trans. Power Syst., vol. 7, no. 1, pp. 106-113, Feb. 1992.

[9] I. Roytelman and S. M. Shahidehpour, "State estimation for electric power distribution system in quasi real-time conditions," IEEE Trans. Power Del., vol. 8, no. 4, pp. 2009-2015, Oct. 1993.

[10] M. E. Baran and A. W. Kelley, "State estimation for real-time monitoring of distribution systems," IEEE Trans. Power Syst., vol. 9, no. 3, pp. 1601-1609, Aug. 1994.

[11] M. E. Baran and A. W. Kelley, "A branch-current-based state estimation method for distribution systems," IEEE Trans. Power Syst., vol. 10, no. 1 , pp. 483-491, Feb. 1995.

[12] C. N. Lu, J. H. Teng, and W.-H. E. Liu, "Distribution system state estimation," IEEE Trans. Power Syst., vol. 10, no. 1, pp. 229-240, Feb. 1995

[13] W.-M. Lin and J.-H. Teng, "Distribution fast decoupled state estimation by measurement pairing," IEE Proc. Gener. Transm. Distrib., vol. 143, no. 1, pp. 43-48, Jan. 1996.

[14] W.-M. Lin and J.-H. Teng, "State estimation for distribution systems with zero-injection constraints," IEEE Trans. Power Syst., vol. 11, no. 1, pp. 518-524, Feb. 1996.

[15] K. Li, "State estimation for power distribution system and measurement impacts," IEEE Trans. Power Syst., vol. 11, no. 2, pp. 911-916, May 1996.

[16] A. K. Ghosh, D. L. Lubkeman, M. J. Downey, and R. H. Jones, "Distribution circuits state estimation using a probabilistic approach," IEEE Trans. Power Syst., vol. 12, no. 1, pp. 45-51, Feb. 1997.

[17] M. K. Celik and W.-H. E. Liu, "A practical distribution state calculation algorithm," in Proc. IEEE PES Winter Meeting, New York, NY, USA, 1999, pp. 442-447.
[18] D. L. Lubkeman, J. Zhang, A. K. Ghosh, and R. H. Jones, "Field results for a distribution circuit state estimator implementation," IEEE Trans. Power Del., vol. 15, no. 1, pp. 399-406, Jan. 2000.

[19] M. E. Baran, "Challenges in state estimation on distribution systems," in Proc. IEEE PES Sum. Meeting, Vancouver, BC, Canada, 2001, pp. 429-433.

[20] H. Wang and N. N. Schulz, "A revised branch current-based distribution system state estimation algorithm and meter placement impact," IEEE Trans. Power Syst., vol. 19, no. 1, pp. 207-213, Feb. 2004

[21] R. Singh, B. C. Pal, and R. A. Jabr, "Choice of estimator for distribution system state estimation," IET Gener. Transm. Distrib., vol. 3, no. 7, pp. 666-678, Jan. 2009.

[22] J. D. Glover, M. S. Sarma, and T. J. Overbye, Power System Analysis and Design, vol. 1, 5th ed. Boston, MA, USA: Cengage Learn., 2010.

[23] G. A. Taylor, M. R. Irving, N. Nusrat, R. Liao, and S. Panchadcharam, "Smart distribution network operation: Emerging techniques and standards," in Proc. IEEE PES Gen. Meeting, Detroit, MI, USA, 2011 pp. 1-6.

[24] N. Deo, Graph Theory With Applications to Engineering and Computer Science, 1st ed. Englewood Cliffs, NJ, USA: Prentice-Hall, 1974.

[25] E. A. Gonzalez, "Introductory graph theory for electrical and electronics engineers," IEEE Multidiscip. Eng. Educ. Mag., vol. 2, no. 2, pp. 5-13, Jun. 2007.

[26] Distribution Testing System of 1481 Buses. [Online]. Available: http://www.feis.unesp.br/Home/departamentos/ engenhariaeletrica/lapsee807/gototheenglishpage/distribution_network_ 1481 lines.rar, accessed Mar. 19, 2015.

[27] J. B. Leite and J. R. S. Mantovani, "Development of a smart grid simulation environment, part I: Project of the electrical devices simulator,' J. Control Autom. Elect. Syst., vol. 26, no. 1, pp. 80-95, Feb. 2015.

Jônatas Boás Leite (S'10) received the B.Sc. and Ph.D. degrees in electrical engineering from São Paulo State University (UNESP)/Ilha Solteira-SP, Ilha Solteira, Brazil, in 2010 and 2015, respectively.

He is currently a Postdoctoral Researcher with the Electrical Engineering Post-Graduate Program, UNESP/Ilha Solteira-SP. His current research interests include planning and control of electric power systems.

José Roberto Sanches Mantovani (M'06) received the B.Sc. degree in electrical engineering from São Paulo State University (UNESP)/Ilha Solteira-SP, Ilha Solteira, Brazil, in 1981, and the M.S. and Ph.D. degrees in electrical engineering from the University of Campinas (UNICAMP)/Campinas-SP Campinas, Brazil, in 1987 and 1995, respectively.

$\mathrm{He}$ is currently a Professor with the Department of Electrical Engineering, UNESP/Ilha Solteira-SP. His current research interests include planning and control of electric power systems. 